\title{
Severe hypoglycemia during pneumocystis pneumonia treatment associated with trimethoprim-sulfamethoxazole use in a patient on peritoneal dialysis
}

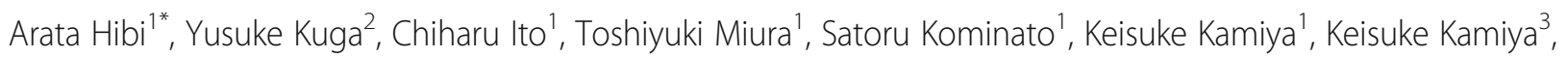
Takahisa Kasugai $^{1}$ and Katsushi Koyama ${ }^{1}$

\begin{abstract}
Background: Trimethoprim-sulfamethoxazole (TMP/SMX) is an essential antimicrobial agent for treating pneumocystis pneumonia (PCP). Furthermore, the risk of hypoglycemia is increased with the co-administration of sulfonylurea due to the presence of the same sulfanilamide structural group in SMX and sulfonylurea. However, hypoglycemia caused by a single administration of TMP/SMX is a rare adverse reaction, and not many cases have been reported. Renal failure is a risk factor for hypoglycemia with single TMP/SMX administration.

Case presentation: A 54-year-old Japanese woman on peritoneal dialysis (PD) for 10 years was admitted to our hospital for the suspicion of PCP. She underwent immunosuppressive treatment with oral prednisolone (3 mg/day) and a subcutaneous injection of adalimumab $(40 \mathrm{mg}$ ) every 2 weeks for rheumatoid arthritis. We initiated the administration of a low-to-moderate dose of TMP/SMX (TMP equivalent to TMP/SMX, approximately $8 \mathrm{mg} / \mathrm{kg} /$ day) in the patient, considering that she was on PD. At 10 days after administration, the patient became unconscious, and blood test results showed that her blood glucose level was low (15 mg/dL). Unlike hypoglycemia, her serum insulin levels were abnormally high $(69.4 \mu \mathrm{U} / \mathrm{mL})$ at that time. Glucose injection was used for correcting the hypoglycemia, but it was refractory. Highly concentrated glucose infusion was needed to maintain her blood glucose level normal. Her serum insulin level was confirmed to have returned to normal $(4.0 \mu \mathrm{U} / \mathrm{mL})$ at 9 days after completing the TMP/SMX treatment.

Conclusions: We suspected that the refractory hypoglycemia in this case was caused by high levels of insulin secretion due to the accumulation of TMP/SMX. One of the risk factors in this patient was the low excretion rate of TMP/SMX into the PD fluid. Although hypoglycemia is a rare complication of TMP/SMX, we should consider this risk during TMP/SMX use in patients, especially those on PD.
\end{abstract}

Keywords: Trimethoprim-sulfamethoxazole, Hypoglycemia, Pneumocystis pneumonia, Peritoneal dialysis

\footnotetext{
* Correspondence: exchange1113@yahoo.co.jp

${ }^{1}$ Division of Nephrology and Rheumatology, Department of Internal

Medicine, Kariya Toyota General Hospital, 5-15, Sumiyoshi-cho, Kariya, Aichi

448-8505, Japan

Full list of author information is available at the end of the article
} 


\section{Background}

Trimethoprim-sulfamethoxazole (TMP/SMX) is a combination of two antimicrobial agents: TMP and SMX. TMP/SMX is used for the prophylaxis of and as a firstline treatment for pneumocystis pneumonia (PCP), which is caused by Pneumocystis jirovecii in human immunodeficiency virus (HIV) patients and non-HIV immunocompromised hosts [1]. PCP is a fatal disease, especially in non-HIV patients. The mortality rate is much higher in non-HIV patients $(50 \%)$ than in HIV patients (6.7\%) [2]. For appropriate treatment, an adequate amount of TMP/SMX is needed. TMP/SMX is an antibiotic that needs dose adjustment in accordance with the patient's renal function.

Hypoglycemia is a rare adverse reaction of TMP/SMX administration. However, co-administration with sulfonylurea increases the risk of hypoglycemia by 6.6 times [3]. Nunnari et al. reported two cases of TMP/SMXassociated severe hypoglycemia, which was caused by a sulfonylurea-like effect of TMP/SMX. They suggested that the risk of hypoglycemia should be considered, especially when prolonged treatment and high dosage is used. SMX is considered to have a sulfonylurea-like effect of increasing endogenous insulin secretion because insulin levels are elevated after administration [4]. The presence of the same sulfanilamide structural group in the two agents (Fig. 1) may be an explanation for this effect. Kenteu et al. reported an acute glycemic effect of TMP/SMX. They performed a 75-g oral glucose tolerance test with or without oral TMP/SMX administration in healthy adults. None of the volunteers experienced hypoglycemia, but their glycemic response was reduced after TMP/SMX administration [5]. This result clinically supports the stimulation of insulin secretion effect of SMX.

Strevel et al. reviewed 13 cases of TMP/SMX-induced hypoglycemia and reported that renal insufficiency is the most prevalent predisposing risk factor (93\%) [6]. Only few cases of hypoglycemia caused by the single administration of TMP/SMX have been reported in Japan $[7,8]$ since the first Japanese case was reported in 1987 [7]. Cases of patients on peritoneal dialysis (PD) have not yet been reported in Japan.
An ideal TMP/SMX dose for treating a PCP patient on $\mathrm{PD}$ has not been described. The risk of hypoglycemia during treatment in a patient on PD should be considered.

\section{Case presentation}

A 54-year-old Japanese woman visited our hospital with the chief complaint of worsening cough and persistent mild fiver. She had been maintained on PD due to endstage renal disease (ESRD) for 10 years. She had anuria and a history of rheumatoid arthritis (RA), for which she underwent immunosuppressive treatment with daily oral prednisolone $(3 \mathrm{mg}$ ) and a subcutaneous injection of adalimumab (40 mg) every 2 weeks. She also had a history of hypertension, chronic heart failure, and osteoporosis. Her other maintenance medications were lansoprazole (10 mg), nifedipine (40 mg), doxazosin (2 mg), carvedilol $(10 \mathrm{mg})$, ferric citrate hydrate $(1000 \mathrm{mg})$, lanthanum carbonate hydrate $(750 \mathrm{mg})$, and calcitriol $(0.30 \mu \mathrm{g})$. Hypoglycemic agents and insulin analogs were not used. She did not have any surgical history except for PD catheter insertion.

Her body weight was $42.6 \mathrm{~kg}$; height, $150.1 \mathrm{~cm}$; and body mass index, $18.9 \mathrm{~kg} / \mathrm{m}^{2}$. She had a mild fever $\left(37.4{ }^{\circ} \mathrm{C}\right.$ ), with blood pressure of $154 / 81 \mathrm{mmHg}$ and tachycardia (heart rate $=100$ beats $/ \mathrm{min}$ ). She had tachypnea (respiratory rate $=20$ breaths $/ \mathrm{min}$ ), and her $\mathrm{SpO}_{2}$ in room air was $99 \%$. On physical examination, a systolic murmur was heard at the apex, but lung auscultation revealed unremarkable findings. Mild ulnar deviations and hallux valgus due to RA were seen, with no pitting edema on the lower extremities. Computed tomography (CT) of the chest showed bilateral ground-glass opacities (Fig 2). Her blood counts were as follows: white blood cells, $8200 / \mu \mathrm{L}$; red blood cells, $352 \times 10^{4} / \mu \mathrm{L}$; hemoglobin, $11.1 \mathrm{~g} / \mathrm{dL}$; and platelets, $26.4 \times 10^{4} / \mu \mathrm{L}$. Her blood glucose was $125 \mathrm{mg} / \mathrm{dL}$; blood urea nitrogen, $58.9 \mathrm{mg} / \mathrm{dL}$; and creatinine, $8.96 \mathrm{mg} / \mathrm{dL}$. Her hemoglobin A1c was 4.5\%. C-reactive protein levels were elevated by $7.23 \mathrm{mg} / \mathrm{dL}$, and $\beta$-D-glucan levels were also high $(23.3 \mathrm{pg} / \mathrm{mL})$. HIV antibody was negative. $P$. jirovecii DNA polymerase chain reaction of sputum was positive. Bronchoscopy was considered but could not be performed due to the patient's refusal. According to these results, a clinical

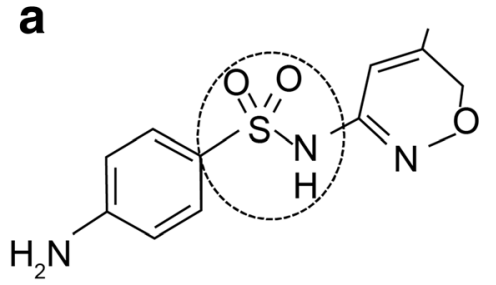

b

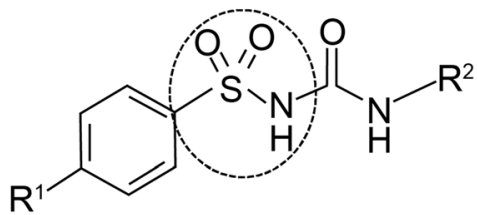

Fig. 1 Structural similarities between sulfamethoxazole (a) and the basic structure of sulfonylurea (b). The sulfonamide group is encircled by the dotted line. $\mathrm{R}^{1}$ and $\mathrm{R}^{2}$ are different in each sulfonylurea 


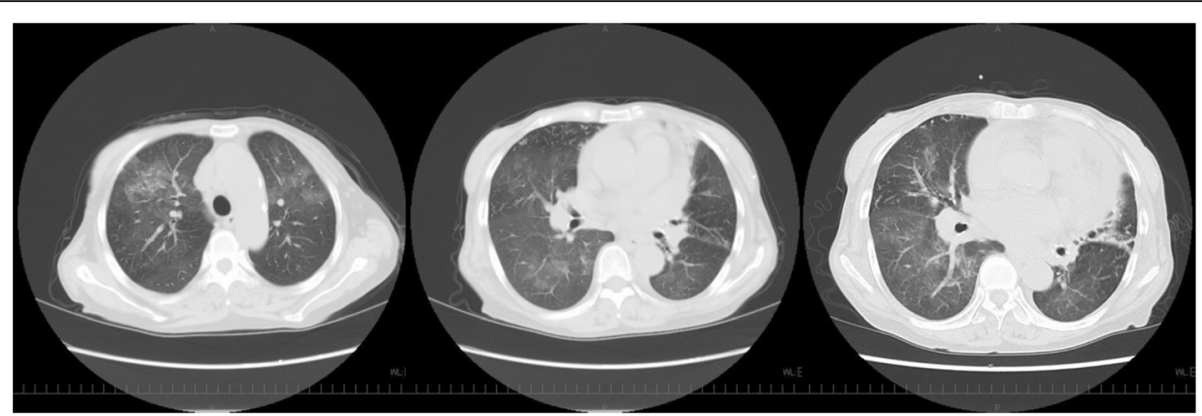

Fig. 2 Computed tomography images taken on the day of admission. Bilateral ground-glass opacities were seen. Pleural effusions, lymphadenopathy, and nodules were not seen

diagnosis of PCP was made. Cytomegalovirus (CMV) pneumonia, tuberculosis, and other fungal infections were differential diagnoses, but CMV antigenemia, T-SPOT, Candida antigen, Aspergillus antigen, and Cryptococcus neoformans antigen were negative.

We started treatment with a low-to-moderate dose of TMP/SMX (TMP equivalent to TMP/SMX, approximately $8 \mathrm{mg} / \mathrm{kg} /$ day), considering that the patient was on PD and was anuric. At 10 days after TMP/SMX administration, the patient suddenly became unconscious after complaining of palpitations and sweating. She was perspiring markedly and did not respond to orders. At that time, her vital signs were as follows: body temperature, $37.0{ }^{\circ} \mathrm{C}$; blood pressure, $140 / 70 \mathrm{mmHg}$; heart rate, 112 beats $/ \mathrm{min}$; and respiratory rate, 24 breaths/min; $\mathrm{SpO}_{2}$ in room air was $99 \%$. Blood glucose levels were immediately checked, and results indicated that the levels were low $(20 \mathrm{mg} / \mathrm{dL})$. Glucose injection (10 g/shot) was used for correcting the hypoglycemia. The glucose levels were temporarily elevated to normal, but they soon went down. Even after 22 glucose injections (220 g of glucose in total) and glucose infusions (75 $\mathrm{g}$ of glucose over $18 \mathrm{~h}$ ), the severe hypoglycemia (15 mg/dL) persisted (Fig. 3). We measured the serum insulin and C-peptide levels during severe hypoglycemia, and results indicated levels of $69.4 \mu \mathrm{U} / \mathrm{mL}$ and $13.10 \mathrm{ng} / \mathrm{mL}$, respectively. Insulin autoimmune syndrome was ruled out because insulin antibody was negative. Adrenal insufficiency was considered because the patient's cortisol level was relatively low at that time

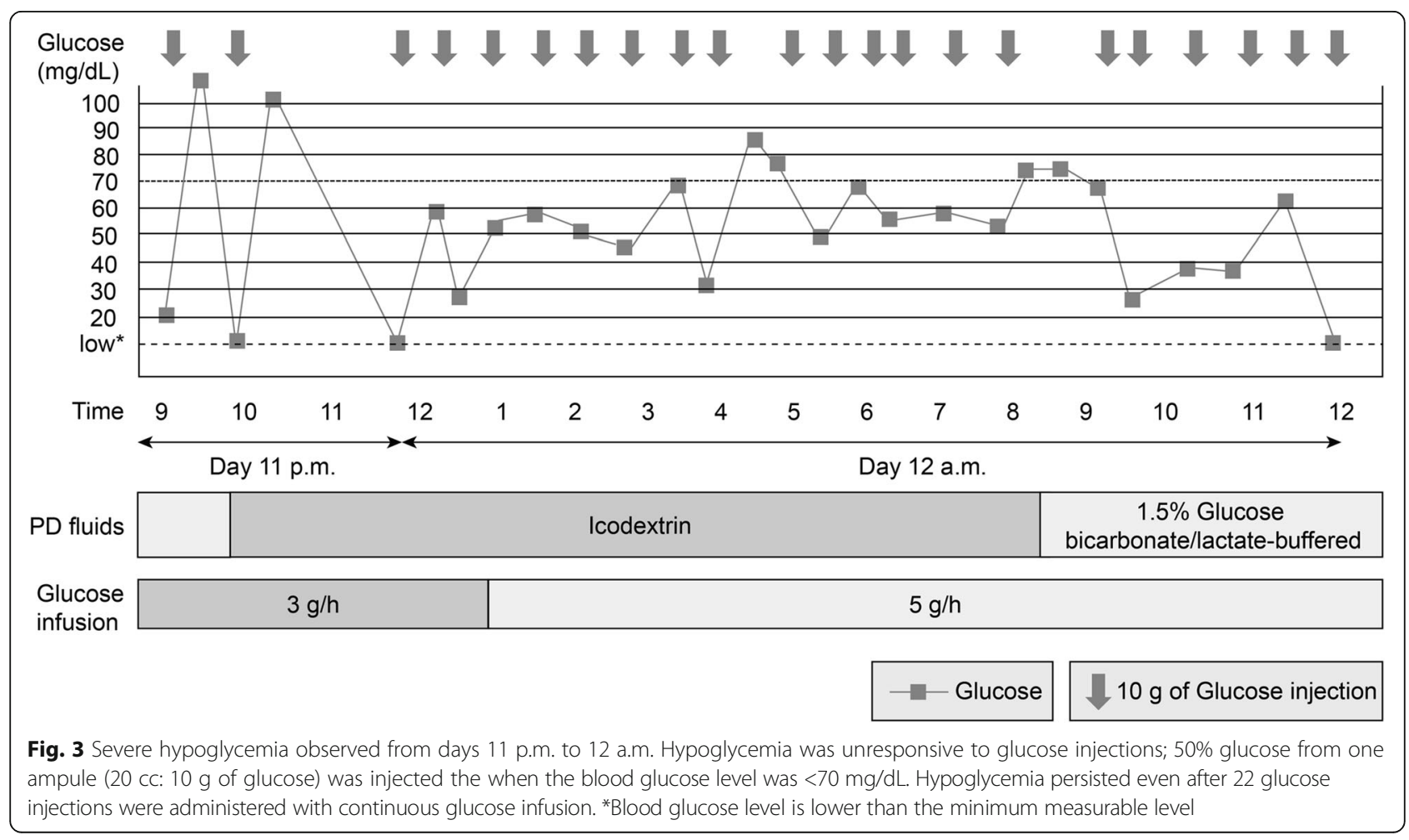


(Table 1), but she was taking oral prednisolone daily, and it could not explain the hyperinsulinemia and persistent severe hypoglycemia that could not be corrected by glucose injections and infusions. Insulinoma was clinically ruled out because the patient had no previous history of hypoglycemia, and the blood glucose levels were unresponsive; in addition, there were no nodules and masses in the pancreas, according to CT images.

We suspected TMP/SMX-induced severe hypoglycemia. Because the patient needed to continue PCP treatment for 14 days, we inserted a central venous catheter in her right neck to infuse highly concentrated glucose $(50 \%$ glucose) and maintain normal blood glucose levels. Using a glucose infusion at $20 \mathrm{~g} / \mathrm{h}$ led to an improvement in the hypoglycemia, but the patient exhibited asymptomatic hypoglycemia at several instances. We decreased the glucose infusion to $10 \mathrm{~g} / \mathrm{h}$ on day 17 (the day that PCP was successfully treated for 14 days with TMP/SMX administration), but the patient had hypoglycemia during early morning. We monitored her blood glucose levels using real-time continuous glucose monitoring (RT-CGM, THE MINIMED ${ }^{\circ}$ 620G SYSTEM, Medtronic Japan Co., Ltd., Tokyo, Japan) and corrected her hypoglycemia by adjusting the amount of glucose infusion (Fig. 4). On day 22, glucose infusion was stopped, and hypoglycemia was no longer detected. After the patient was stabilized, we measured her serum insulin levels, which had returned to normal $(4.0 \mu \mathrm{U} / \mathrm{mL}$ on day 23). This result supported a definitive diagnosis of TMP/ SMX-associated severe hypoglycemia. On day 23 , an adrenocorticotropic hormone stimulation test-measuring serum cortisol levels immediately before as well as 30 and $60 \mathrm{~min}$ after the intravenous injection of $250 \mu \mathrm{g}$ of cosyntropin-was performed, but the results indicated unresponsiveness (data not shown). This finding is compatible with tertiary adrenal insufficiency due to long-term steroid use.

We added a low-dose of TMP/SMX (TMP equivalent to TMP/SMX, $40 \mathrm{mg}$ ) in this patient for prophylaxis against $\mathrm{PCP}$ because she had a high risk of recurrent $P$. jirovecii infection. After taking a low dose of TMP/SMX, the patient did not become hypoglycemic and was discharged on day 36 without any medical complications.

\section{Discussion}

TMP/SMX is well-absorbed through oral administration and has a good bioavailability. Accumulation of the drug should be considered in a patient with compromised renal function due to the prolongation of its half-life [9]. The structural similarity of SMX with sulfonylurea may result in pancreatic insulin secretion, particularly at higher doses and in patients with renal impairment [6]. We reviewed previously reported 22 cases of TMP/ SMX-associated hypoglycemia [4, 6-8, 10-25] (Table 2). In most of the cases (77\%), patients had renal impairment (serum creatinine $>1.1 \mathrm{mg} / \mathrm{dL}$ and/or on renal replacement therapy). A moderate-to-high dose of TMP/SMX ( $\geq 1600 \mathrm{mg}$ of SMX/ day) was used for treating PCP in $45 \%$ of the cases. We could find two cases of HD patients $[11,14]$ and one case of PD patient [16]. Although serum insulin levels were not available for all the cases, in most cases, hyperinsulinemia was observed despite low serum blood glucose levels. We considered that hyperinsulinemia was caused by insulin secretion due to the sulfonylurea-like effect of SMX, and renal impairment and high-dose TMP/SMX use were two major predisposing factors for TMP/SMX-induced hypoglycemia. The kidneys have an important role in the clearance and degradation of circulating insulin. It is estimated that $30-80 \%$ of insulin entering the kidneys is removed [26]. Although conclusive evidence regarding the contribution of the kidneys to total body gluconeogenesis is not available, the kidneys contribute to the maintenance of glucose homeostasis to some extent [27]. Similar to our case, we should consider a prolonged insulin effect and low ability to maintain glucose homeostasis in ESRD patients without urine output.

We suspected that the persistent hypoglycemia in the present case was caused by the accumulation of SMX in this patient because of PD without urine output. Walker et al. reported the pharmacokinetics of TMP/SMX in 10 adult patients on continuous ambulatory PD. The patients were given one dose of TMP (320 mg) and SMX (1600 mg) orally, intravenously, and intraperitoneally on separate occasions. At $24 \mathrm{~h}$ after administration, $<3 \%$ of TMP and $<6 \%$ of SMX were excreted in the dialysate,

Table 1 Laboratory data on days 11 and 23 (blood samples were taken early morning)

\begin{tabular}{lll}
\hline & Day 11 (10 days after TMP/SMX administration) & Day 23 (9 days after completing TMP/SMX treatment) \\
\hline Glucose & $15 \mathrm{mg} / \mathrm{dL}$ & $73 \mathrm{mg} / \mathrm{dL}$ \\
Serum insulin & $69.4 \mu \mathrm{U} / \mathrm{mL}$ & $4.0 \mu \mathrm{U} / \mathrm{mL}$ \\
Serum C-peptide & $13.10 \mathrm{ng} / \mathrm{mL}$ & $2.07 \mathrm{ng} / \mathrm{mL}$ \\
Serum cortisol & $7.2 \mu \mathrm{g} / \mathrm{dL}$ & $3.1 \mu \mathrm{g} / \mathrm{dL}$ \\
ACTH & $75.9 \mathrm{pg} / \mathrm{mL}$ & $28.2 \mathrm{pg} / \mathrm{mL}$ \\
Anti-insulin antibody & Negative & \\
\hline
\end{tabular}

TMP/SMX trimethoprim-sulfamethoxazole, $A C T H$ adrenocorticotropic hormone 


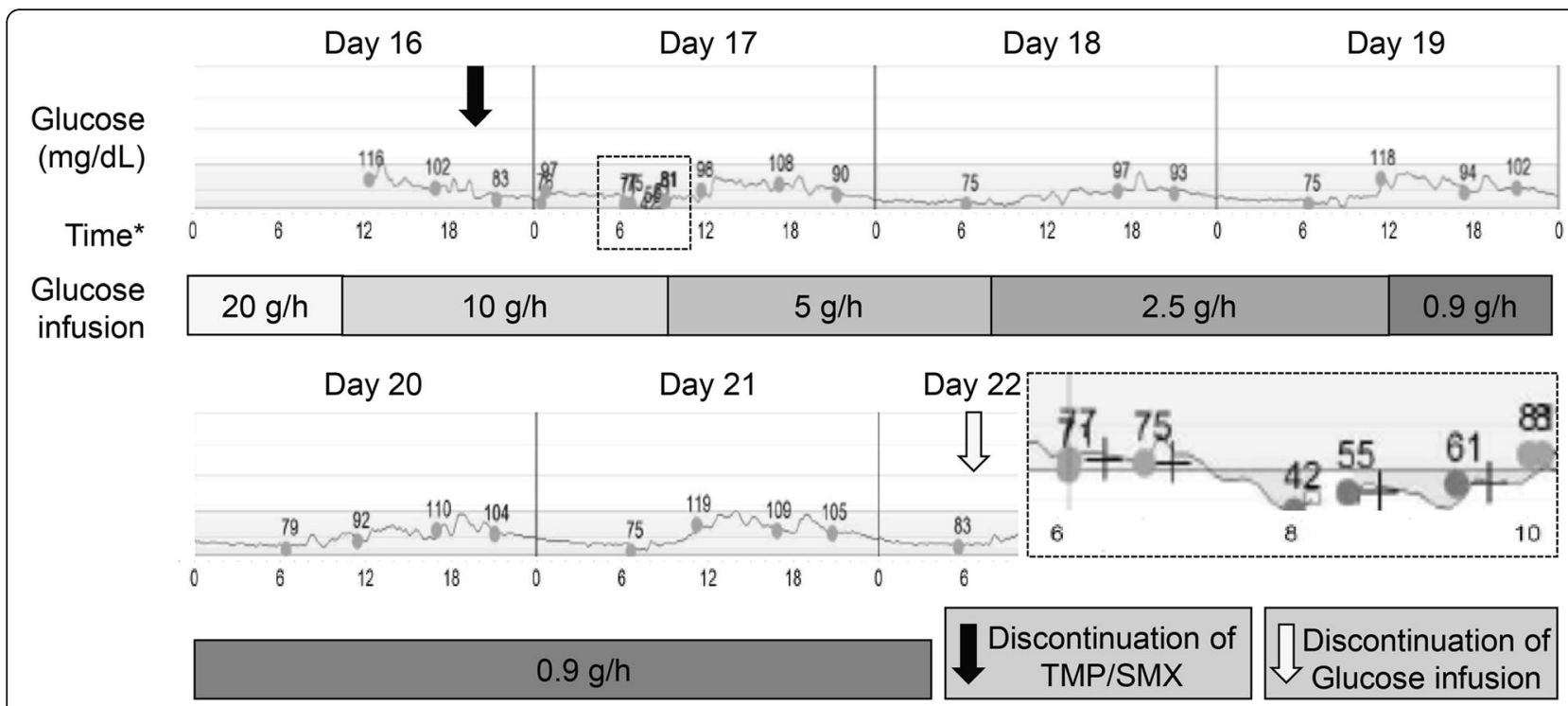

Fig. 4 RT-CGM results from days 16 to 21. The patient exhibited hypoglycemia again on the morning of day 17 (boxed by the dotted line, magnified on the lower right). The amount of glucose infusion was reduced by gradually adjusting the injection rate of $50 \%$ glucose fluid. *Time is written as military time (commonly used in Japan). RT-CGM: real-time continuous glucose monitoring, TMP/SMX: trimethoprim-sulfamethoxazole

regardless of the route of administration [28]. To achieve prompt recovery, hemodialysis (HD) might be a better choice because HD has a higher clearance of SMX compared with CAPD. Nissenson et al. reported the pharmacokinetics of intravenous TMP/SMX during HD. A total of 16 patients with ESRD received a single ampule of TMP/SMX (containing $800 \mathrm{mg}$ of SMX) at $45 \mathrm{~min}$ prior to HD, and $57 \%$ of the administered SMX was removed during the 4-h HD [29].

In this case, long-term steroid use was also a risk for prolonged hypoglycemia due to tertiary adrenal insufficiency, because steroids suppress the secretion of corticotropin-releasing hormone from the hypothalamus [30]. Senanayake et al. reported a case of hypoglycemia caused by a prophylactic dose of TMP/SMX in a patient with eosinophilic granulomatosis with polyangiitis using prednisolone for immunosuppressive therapy, although the patient was without renal impairment [24]. Symptoms of hypoglycemia were ameliorated by increasing the prednisolone dosage. In our case, increasing the steroid dosage could have been considered to reduce the amount of glucose infusions because the patient had anuria. Volume overload due to infusion becomes a serious problem in PD patients because the rehydration amount is limited in these patients.

There was a possibility that the glucose in the PD fluid acted as a buffer against hypoglycemia to some extent in this case. The patient used icodextrin PD fluid during the night time (from 10 p.m. to 9 a.m., $2000 \mathrm{~mL}$ ) and $1.5 \%$ glucose bicarbonate/lactate-buffered PD fluid from
9 a.m. to 10 p.m. (three cycles of $2000 \mathrm{~mL}$ ). Icodextrin use might be a part of the reason why hypoglycemia was seen mainly from midnight to early morning in this case.

Although RT-CGM is usually used for monitoring the glucose levels of patients with type 1 diabetes [31], it was effective in this case to detect asymptomatic hypoglycemia earlier, and it enabled the patient to monitor glucose levels more appropriately compared with the self-monitoring of blood glucose. Lower dose TMP/SMX for prophylaxis against PCP was acceptable in this patient, along with the careful monitoring of blood glucose levels after the detachment of RT-CGM during hospitalization.

\section{Conclusions}

We encountered a complicated case of severe hypoglycemia associated with TMP/SMX use in a patient on PD. To our knowledge, this is the first Japanese case of hypoglycemia in a patient on PD caused by TMP/SMX use alone. Hypoglycemia was persistent and refractory, and glucose infusion was needed for almost a week even after PCP treatment was completed to maintain the blood glucose at normal levels. Although hypoglycemia is a rare adverse reaction when TMP/SMX is used in isolation, the risk of hypoglycemia should be considered when using higher than average doses of TMP/SMX in patients with renal failure, especially in those on $\mathrm{PD}$, because of the lower excretion rate of the drug into PD fluids. Routine blood glucose monitoring might be recommended in selected cases. 


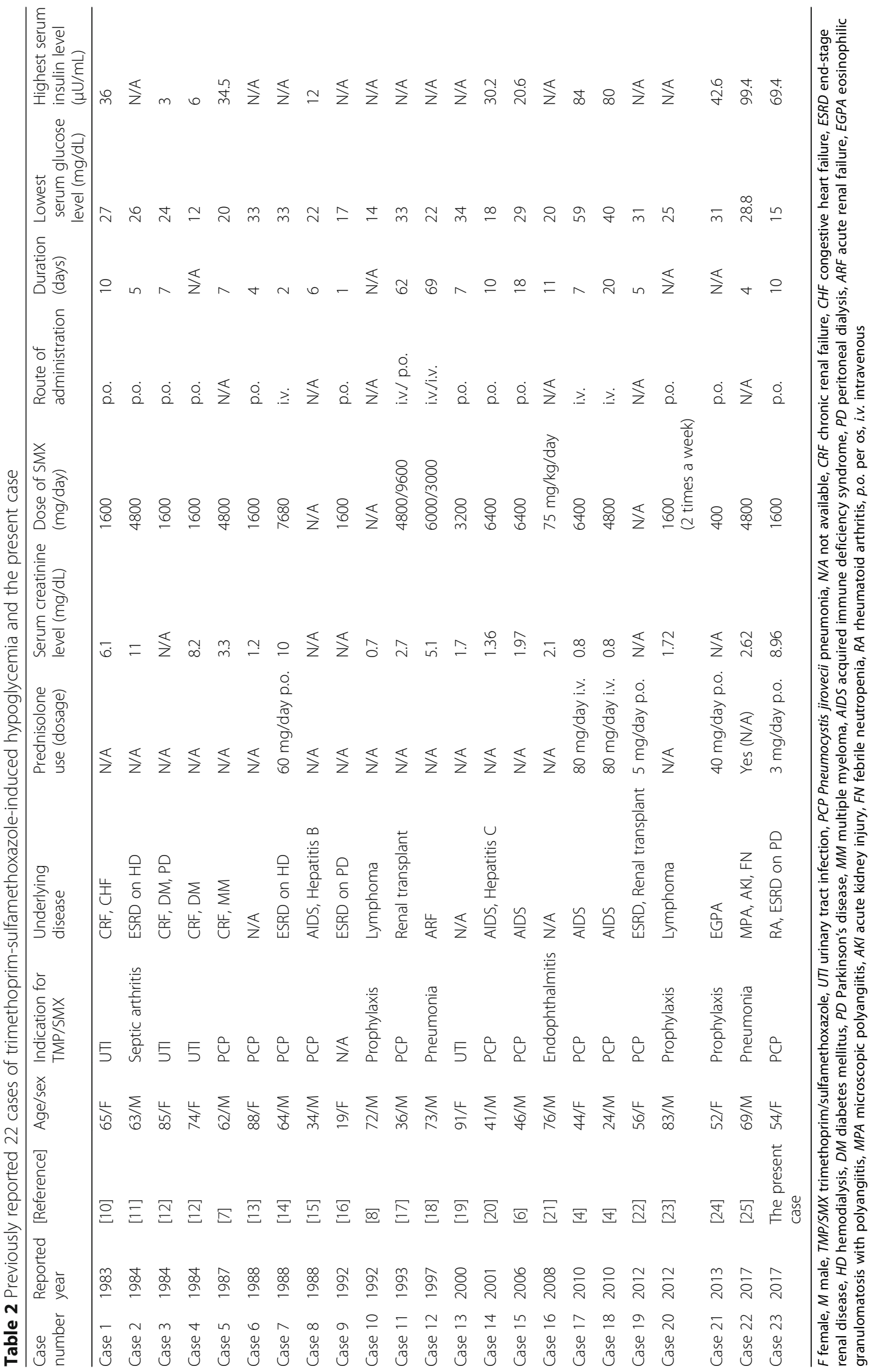




\section{Abbreviations}

CMV: Cytomegalovirus; CT: Computed tomography; ESRD: End-stage renal disease; HD: Hemodialysis; PCP: Pneumocystis pneumonia; PD: Peritoneal dialysis; RA: Rheumatoid arthritis; RT-CGM: Real-time continuous glucose monitoring; SMX: Sulfamethoxazole; TMP: Trimethoprim

\section{Acknowledgements}

We are grateful to Saori Minami, an attentive nurse who reported sudden changes in the patient and checked her blood glucose levels immediately to make a correct diagnosis promptly.

\section{Funding}

The authors declare that there is no funding related to this manuscript.

\section{Availability of data and materials}

Data will be made available by the corresponding author upon request.

\section{Authors' contributions}

$\mathrm{AH}$ is responsible for the manuscript. MT, KY, and $\mathrm{KK}$ contributed to the critical revision of the manuscript. KY contributed to the interpretation of endocrinological test results. TK, Cl, and SK contributed to the interpretation of other test results. All authors read and approved the final version of the manuscript.

\section{Ethics approval and consent to participate}

According to the Ethical Guidelines for Medical and Health Research involving Human Subjects in Japan, ethics approval is not necessary for case reports.

\section{Consent for publication}

Written informed consent was obtained from the patient for the publication of this case report and any accompanying test results.

\section{Competing interests}

The authors declare that they have no competing interests.

\section{Publisher's Note}

Springer Nature remains neutral with regard to jurisdictional claims in published maps and institutional affiliations.

\section{Author details}

'Division of Nephrology and Rheumatology, Department of Internal Medicine, Kariya Toyota General Hospital, 5-15, Sumiyoshi-cho, Kariya, Aichi 448-8505, Japan. ${ }^{2}$ Division of Endocrinology and Metabolism, Department of Internal Medicine, Kariya Toyota General Hospital, 5-15, Sumiyoshi-cho, Kariya, Aichi 448-8505, Japan. ${ }^{3}$ Division of Nephrology and Rheumatology, Department of Internal Medicine, Aichi Medical University Hospital, 1-1 Yazakokarimata, Nagakute, Aichi 480-1195, Japan.

Received: 15 June 2017 Accepted: 10 August 2017

Published online: 02 November 2017

\section{References}

1. Catherinot $E$, Lanternier $F$, Bougnoux ME, Lecuit $M$, Couderc $L$, Lortholary O. Pneumocystis jirovecci Pneumonia. Infect Dis Clin Am. 2010;24:107-38.

2. Su YS, Lu JJ, Peng CL, Chang FY. Pneumocystis jirovecii pneumonia in patients with and without human immunodeficiency virus infection. J Microbiol Immunol Infect. 2008;41:472-82.

3. Juurlink DN, Mamdani M, Kopp A, Laupacis A, Redelmeier DA. Drug-drug interactions among elderly patients hospitalized for drug toxicity. JAMA. 2003;289:1652-8

4. Nunnari G, Celesia BM, Bellissimo F, Tosto S, La Rocca M, Giarratana F, et al. Trimethoprim-sulfamethoxazole-associated severe hypoglycaemia: a sulfonylurea-like effect. Eur Rev Med Pharmacolo Sci. 2010;12:1015-8.

5. Kenteu B, Noubiap JJ, Etoa MC, Azabji-Kenfack M, Dehayem M, Sobngwi E. Acute glycaemic effects of co-trimoxazole at prophylactic dose in healthy adults. BMC Endocr Disord. 2016;16:62

6. Strevel EL, Kuper A, Gold WL. Severe and protracted hypoglycaemia associated with co-trimoxazole use. Lancet Infect Dis. 2006;6:178-82.
7. Fukuda H, Ohiwa T, Kusano Y, Ohbu S, Niikuma H, Terada H. A case of hypoglycemic attack associated with sulfamethoxazole-trimethoprim. J Jpn Soc Int Med. 1987;76:1887-8. [in Japanese]

8. Yamawaki Y. Hypoglycemic attack which seems to be due to ST combination. A case of repeated hypoglycemia during the course of malignant lymphoma. J-ARIS. 1992;5:996-1000. (in Japanese)

9. Paap CM, Nahata MC. Clinical use of trimethoprim/sulfamethoxazole during renal dysfunction. DICP. 1989;23:646-54

10. Arem R, Garber AJ, Field JB. Sulfonamide-induced hypoglycemia in chronic renal failure. Arch Intern Med. 1983:4:827-9.

11. Frankel MC, Leslie BR, Sax FL, Soave R. Trimethoprim-sulfamethoxazole-related hypoglycemia in a patient with renal failure. N Y State J Med. 1984;1:30-1.

12. Poretsky L, Moses AC. Hypoglycemia associated with trimethoprim/ sulfamethoxazole therapy. Diabetes Care. 1984;5:508-9.

13. McKnight JT, Gaskins SE, Pieroni RE, Machen GM. Sever hypoglycemia associated with trimethoprim-sulfamethoxazole therapy. J Am Board Fam Pract. 1988:2:143-5.

14. Ryan DW, Oyston J. Sulphonylureas and hypoglycaemia. Br Med J (Clin Res Ed). 1988:6632:1328.

15. Schattner A, Rimon E, Green L, Coslovsky R, Bentwich Z. Hypoglycemia induced by co-trimoxazole in AIDS. BMJ. 1988;6650:742.

16. Mansoor GA, Nicholson GD. Hypoglycemia in chronic renal failure. West Indian Med J. 1992;1:41-2.

17. Johnson JA, Kappel JE, Sharif MN. Hypoglycemia secondary to trimethoprim/ sulfamethoxazole administration in a renal transplant patient. Ann Pharmacother. 1993;3:304-6.

18. Lee AJ, Maddix DS. Trimethoprim/sulfamethoxazole-induced hypoglycemia in a patient with acute renal failure. Ann Pharmacother. 1997:6:727-32.

19. Mathews WA, Manint JE, Kleiss J. Trimethoprim-sulfamethoxazole-induced hypoglycemia as a cause of altered mental status in an elderly patient. J Am Board Fam Pract. 2000:3:211-2.

20. Hughes CA, Chik CL, Taylor GD. Co-trimoxazole-induced hypoglycemia in an HIV-infected patient. Can J Infect Dis. 2001:5:314-6.

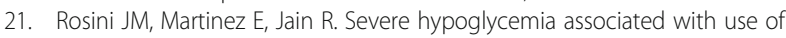
trimethoprim/sulfamethoxazole in a patient with chronic renal insufficiency. Ann Pharmacother. 2008;4:593-4.

22. Forde DG, Aberdein J, Tunbridge A, Stone B. Hypoglycemia associated with co-trimoxazole use in a 56-year-old Caucasian woman with renal impairment. BMJ Case Rep. 2012; doi:10.1136/bcr-2012-007215.

23. Caro J, Navarro-Hidalgo I, Civera M, Real JT, Ascaso JF. Severe, long-term hypoglycemia induced by co-trimoxazole in a patient with predisposing factors. Endocrinol Nutr. 2012:2:146-8.

24. Senanayake R, Mukhtar M. Co-trimoxazole-induced hypoglycemia in a patient with churg-strauss syndrome. Case Rep Endocrinol. 2013: doi:10.1155/2013/415810

25. Conley TE, Mohiuddin A, Naz N. Sever co-trimoxazole-induced hypoglycemia in a patient with microscopic polyangiitis. BMC Case Rep. 2017; doi:10.1136/ brc-2016-218976. 25

26. Rabkin R, Ryan MP, Duckworth WC. The renal metabolism of insulin. Diabetologia. 1984;3:351-7.

27. Triplitt CL. Understanding the kidneys' role in blood glucose regulation. Am J Manag Care. 2012;18(Suppl 1):11-6.

28. Walker SE, Paton TW, Churchill DN, Ojo B, Manuel MA, Wright N. Trimethoprim sulfamethoxazole pharmacokinetics during continuous ambulatory peritoneal dialysis (CAPD). Perit Dial Int. 1989:9:51-5.

29. Nissenson AR, Wilson C, Holazo A. Pharmacokinetics of intravenous trimethoprim-sulfhamethoxazole during hemodialysis. Am J Nephrol. 1987;7:270-4

30. Charmandari E, Nicolaides NC, Chrousos GP. Adrenal insufficiency. Lancet 2014;383:2152-67. doi:10.1016/s0140-6736(13)61684-0.

31. Choudhary P, Ramasamy S, Green L, Gallen G, Pender S, Brackenridge A Amiel SA, Pickup JC. Real-time continuous glucose monitoring significantly reduces severe hypoglycemia in hypoglycemia-unaware patients with type 1 diabetes. Diabetes Care. 2013:36:4160-2. 